\title{
Lower Bounds on the Vertex Cover Number and Energy of Graphs
}

\author{
Farzad Shaveisi \\ Department of Mathematics, Faculty of Science, Razi University, Kermanshah, Iran \\ f.shaveisi@razi.ac.ir
}

(Received September 6, 2021)

\begin{abstract}
Let $G$ be a graph with $n$ vertices and $m$ edges. A vertex cover of $G$ is a set $Q$ of vertices that contains one endpoint of every edge; the minimum cardinality of a vertex cover is called the vertex cover number and it is denoted by $\beta$. In this paper, we prove new lower bounds for the vertex cover number. For example it is shown that $\beta \geq \frac{n}{\Delta+1}$ and $\beta \geq \frac{\sqrt{(2 \Delta-1)^{2}+8 m}-(2 \Delta-1)}{2}$, where $\Delta$ denotes the maximum degree of $G$. Moreover, both of these inequalities turn into equalities if and only if $G$ is a star graph. Some new lower bounds for the energy of $G$ are given, too.
\end{abstract}

\section{Introduction}

Let $G=(V, E)$ be a simple graph with vertex set $V=\left\{v_{1}, v_{2}, \ldots, v_{n}\right\}$ and the edge set $E$. The degree of a vertex $x$ of $G$ is denoted by $d(x)$ and the maximum (resp. minimum) degree of vertices of $G$ is denoted by $\Delta=\Delta(G)$ (resp. $\delta=\delta(G)$ ). Avertex in $G$ is said to be isolated vertex if it has degree zero. For any graph $G$ with $n$ vertices and $m$ edges, $m-n+1$ is called the cyclomatic number of $G$. In this paper, the number of connected components (maximal connected subgraphs) of $G$ is denoted by $c(G)$. Also, the adjacency matrix $A(G)=\left(a_{i j}\right)$ is defined as $n \times n$ matrix, where $a_{i j}=1$, if $v_{i}$ is adjacent to $v_{j}$; and $a_{i j}=0$, otherwise. For any matrix $A, A^{*}$ is the conjugate transpose of $A$. A Hermitian matrix is a complex square matrix such $A=A^{*}$. The singular values of a matrix $A$ are defined as the square roots of the eigenvalues of $A^{*} A$, and the energy of $A$ which is denoted by $\mathcal{E}(A)$ is the sum of its singular values. The energy of a graph $G$, 
denoted by $\mathcal{E}(G)$, is defined as the energy of $A(G)$. Note that since $A(G)$ is symmetric real matrix, $\mathcal{E}(G)$ equals the sum of the absolute values of all eigenvalues of $A(G)$. The concept of graph energy was first introduced by Gutman in 1978 [13]. For detailed results on graph energy we refer the reader to book [18], where the authors summarized the most important results involving graph energy. A bipartite graph is a graph whose vertices can be divided into two disjoint parts $U$ and $V$ such that every edge joins a vertex in $U$ to one in $V$. In this paper, the number of odd cycles of the graph $G$ is denoted by $c_{o}(G)$. It is well-known that a graph $G$ is bipartite if and only if $c_{o}(G)=0$. A complete bipartite graph is a bipartite graph in which every vertex of one part is joined to every vertex of the other part. A complete bipartite graph whose size of parts equals $a$ and $b$ is denoted by $K_{a, b}$. If the size of one of the parts is 1 , then it is said to be a star graph. If $X$ is a set of vertices of $G$, then $G[X]$ will denote the subgraph of induced by $X$, and $G-X$ will denote the subgraph of $G$ induced by $V(G) \backslash X$. Throughout this paper, the term $k$-connected means $k$-vertex-connected. For more details about basic notations and definitions, see [5, 10,25].

A vertex cover of a graph $G$ is a set $Q \subset V(G)$ that contains one endpoint of every edge. The vertices in $Q$ cover $E(G)$. The minimum size of a vertex cover of $G$ is called the vertex cover number and it is denoted by $\beta=\beta(G)$. The vertex covering problem and vertex cover number is one of the most studied simple graph invariants; for more studies about this topic, see $[6,11,14,16,17]$. In this paper, we prove some lower bounds for the vertex cover number and the energy of graph depending on number of vertices $n$, number of edges $m$ and maximum degree $\Delta$.

In Section 2, we use the double counting principle to prove that

$$
\beta \geq \frac{n}{\Delta+1}
$$

with equality if and only if $G$ is a star graph. Also, it is shown that if $G$ contains a minimum vertex cover whose induced subgraph is connected, then $\beta \geq \frac{n-2}{\Delta-1}$.

In Section 3, a new lower bound

$$
\beta \geq \sqrt{(2 \Delta-1)^{2}+8 m}-(2 \Delta-1)
$$

is found; also, it is proved that the equality holds for star graphs. Then this lower bound is used to give a lower bound for the energy of graph. Caporossi et al. gave the following lower bound based on the number of edges in [4] as

$$
\mathcal{E}(G) \geq 2 \sqrt{m}
$$


with equality if and only if $G$ is the union of a complete bipartite graph $K_{a, b}$, where $a b=m$, and arbitrarily many isolated vertices. Another lower bound was established in [23] as follows:

$$
\mathcal{E}(G) \geq \sqrt{2 m+n(n-1)|\operatorname{det} A(G)|^{\frac{2}{n}}}
$$

This lower bound was improved in [12] as

$$
\mathcal{E}(G) \geq \sqrt{2 m+n(n-2)|\operatorname{det} A(G)|^{\frac{2}{n}}}
$$

To see more recent bounds for the energy of a graph, see [1-3, 7, 9, 15]. Most of these bounds depends on the linear algebra parameters. In this paper, we give an upper bound for the energy of graph in terms of the number of edges and maximum degree. Then in this section, after recalling some bounds and spectral characterizations for the number of odd cycles $c_{o}(G)$, two lower bounds for the energy of graphs in terms of the number of odd cycles and the maximum degree are given.

\section{New lower bounds for vertex cover number in terms of order and maximum degree}

We start this section by the following result in which a lower bound for the vertex cover number is determined and it is shown that the equality holds only for star graphs.

Theorem 1. Let $G$ be a connected graph with $n$ vertices, $m$ edges, vertex cover number $\beta$ and maximum degree $\Delta \geq 2$. Then

$$
\beta \geq \frac{n}{\Delta+1}
$$

Also, the equality holds if and only if $G$ is a star graph.

Proof. Let $Q$ be a minimum vertex cover of $G$ and let $H=G[Q]$ and $K=G \backslash H$. Let $F$ be the set of edges between $H$ and $K$. Applying the double counting principle, we count the set $F$. Since the vertices in $Q$ cover $E(G), K$ contains no edge and $G$ contains no isolated verex, so

$$
|F| \geq|K|=n-\beta
$$

On the other hand,

$$
|F|=\sum_{v \in Q}\left(d_{G}(v)-d_{H}(v)\right)=\sum_{v \in Q} d_{G}(v)-\sum_{v \in Q} d_{H}(v)=\sum_{v \in Q} d_{G}(v)-2 m(H) \leq \beta \Delta .
$$


Combining (2) with (3), we have

$$
\beta \geq \frac{n}{\Delta+1}
$$

Also, it is clear that $\beta=\frac{n}{\Delta+1}$ if and only if all the following statements are true:

(i) For any vertex $v \in V(H), d(v)=\Delta$, since the equality in (3) holds;

(ii) $H$ is a null graph, i.e. $Q$ is an independent set;

(iii) $|F|=n-\gamma$, i.e. each vertex of $K$ is adjacent to exactly one vertex of $H$, for the equality in (2) holds.

Now, since the complement of any minimum vertex cover is an independent set, we deduce that $G$ satisfies Conditions (i), (ii) and (iii) if and only if $G \cong \beta K_{1, \Delta}$. Therefore the connectivity of $G$ implies that $\beta=\frac{n}{\Delta+1}$ if and only if $G \cong K_{1, \Delta}$.

In the next theorem, the following notation will be used.

$$
c v(G)=\min \{c(G[Q]): Q \text { is a minimum vertex cover of } G\} .
$$

Theorem 2. Let $G$ be a connected graph with order $n$, vertex cover number $\beta$ and maximum degree $\Delta \geq 2$. Then $\beta \geq \frac{n-2 c v(G)}{\Delta-1}$.

Proof. Choose any minimum vertex cover $Q$ of $G$ and let $H=G[X]$ and $K=G \backslash H$.

Let $F$ be the set of edges between $H$ and $K$. Now, we apply the double counting principle, for the set $F$. Since $Q$ is a vertex cover,

$$
|F| \geq|K|=n-\beta
$$

On the other hand,

$$
\begin{aligned}
|F|=\sum_{v \in Q}\left(d_{G}(v)-d_{H}(v)\right) & =\sum_{v \in Q} d_{G}(v)-\sum_{v \in X} d_{H}(v) \leq \beta \Delta-2 m(H) \\
& \leq \beta \Delta-2(\beta-c(H))=\beta(\Delta-2)+2 c(H) .
\end{aligned}
$$

Combining (5) with (6), we have

$$
\beta \geq \frac{n-2 c(H)}{\Delta-1}
$$

Now, let $H$ be induced subgraph on a minimum dominating set such that $c(H)=c v(G)$. Then we have

$$
\beta \geq \frac{n-2 c v(G)}{\Delta-1}
$$

as desired. 
Corollary 3. Let $G$ be a connected graph with order $n$, vertex cover number $\beta$ and maximum degree $\Delta \geq 2$. If $G$ contains a minimum vertex cover $Q$ with $G[Q]$ connected, then $\beta \geq \frac{n-2}{\Delta-1}$.

Proof. Choose a minimum vertex cover $Q$ such that $H=G[Q]$ be connected. Then the assertion follows from (8).

Remark 4. Let $G$ be a graph which contains a minimum dominating set $Q$ with $G[Q]$ connected. Then it is not hard to check that the lower bound, obtained in Corollary 3 is better than the one which was obtained in Theorem 1.

\section{A lower bound for vertex cover number and energy of graphs}

This section is devoted to proving a new lower bound for the vertex cover number depending on the number of edges and maximum degree. Some examples and applications are given, too.

Theorem 5. Let $G$ be a connected graph with $m$ edges, vertex cover number $\beta$ and maximum degree $\Delta \geq 2$. Then

$$
\beta \geq \frac{\sqrt{(2 \Delta-1)^{2}+8 m}-(2 \Delta-1)}{2}
$$

Moreover, the only extremal graph whose vertex cover number attain the lower bound is a star graph.

Proof. Let $Q$ be a minimum vertex cover of $G, H=G[Q]$ and $K=G \backslash H$. We use double counting principle for the set $E(H)$. It is clear that

$$
|E(H)| \leq \frac{\beta(\beta-1)}{2}
$$

On the other hand, since $Q$ is a vertex cover of $G, K$ contains no edge and $G$ contains no isolated verex, so

$$
|E(H)|=m-|F|
$$

Similar to the proof in Theorem 1, we have

$$
|F|=\sum_{v \in Q}\left(d_{G}(v)-d_{H}(v)\right)=\sum_{v \in Q} d_{G}(v)-\sum_{v \in Q} d_{H}(v)=\sum_{v \in Q} d_{G}(v)-2 m(H) \leq \Delta \beta .
$$


Now, from (11) and (12), we obtain the following inequality:

$$
|E(H)| \geq m-\Delta \beta
$$

Combining (10) with (13), we have

$$
\beta^{2}+(2 \Delta-1) \beta-2 m \geq 0 .
$$

Therefore, either $\beta \leq \frac{1-2 \Delta-\sqrt{(2 \Delta-1)^{2}+8 m}}{2}$ or $\beta \geq \frac{1-2 \Delta+\sqrt{(2 \Delta-1)^{2}+8 m}}{2}$. Since $\beta \geq 1$, the first inequality does not hold. So, the assertion follows. Moreover, the equality in (9) holds if and only if all inequalities involved in (10)-(14) turn into equalities, which implies that:

(i) $H$ is a complete graph with $\beta$ vertices, by (10);

(ii) Every vertex in $Q$ is adjacent to $\Delta$ vertices from $K$ and $m(H)=0$, by (12).

Therefore, the equality in (9) holds if and only if $G \cong K_{1, \Delta}$.

Corollary 6. For any connected graph $G$ with $n$ vertices, $m$ edges, maximum degree $\Delta$,

$$
\beta(G) \geq \max \left\{\left\lceil\frac{n}{\Delta+1}\right\rceil,\left\lceil\frac{1-2 \Delta+\sqrt{(2 \Delta-1)^{2}+8 m}}{2}\right\rceil\right\} .
$$

Example 7. Let $G$ be a graph with $n$ vertices, $m$ edges and maximum degree $\Delta$.

(a) If $G \cong K_{1, r}$, then $\beta(G)$ and both lower bounds in (1) and (9) are 1 .

(b) If $G \cong K_{2, r}$, then $\beta(G)=2$ and

$$
\left\lceil\frac{n}{\Delta+1}\right\rceil=\left\lceil\frac{1-2 \Delta+\sqrt{(2 \Delta-1)^{2}+8 m}}{2}\right\rceil=2 .
$$

(c) If $G \cong K_{3,3}$, then $\beta(G)=3$ and

$$
\left\lceil\frac{n}{\Delta+1}\right\rceil=2 ; \quad\left\lceil\frac{1-2 \Delta+\sqrt{(2 \Delta-1)^{2}+8 m}}{2}\right\rceil=3 .
$$

So, for this graph the lower bound (9) is better than that of (1).

In the continuing of this section, we give some upper bounds for the energy of the graph $G$ in terms of its vertex cover number $\beta(G)$ and the number of odd cycles $c_{o}(G)$. Before this, it is needed to recall some bounds and spectral characterizations for the parameter 
$c(G)$. From classical graph theory, we know that a graph is bipartite if and only if it has no odd cycle. If we focus on nonbipartite graphs, then two problems (about odd cycles in graphs), arise.

The first one is about the length and the number of the shortest odd cycles. This problem has been solved by using tools of spectral graph theory. In algebraic graph theory, it is shown that if $\chi(G, \lambda)=\lambda^{n}+a_{1} \lambda^{n-1}+a_{2} \lambda^{n-2}+\cdots+a_{n}$ is the characteristic polynomial of $G$, then the length of the shortest odd cycle in $G$ is equal to the index of the first non-vanishing coefficient among $a_{1}, a_{3}, a_{5}, \ldots$; moreover, the number of such cycles in $G$ equals $-\frac{a_{f}}{2}$. There are many other spectral conditions for odd cycles in non-bipartite graphs. For example, see $[8,19,20]$. Let $S_{2 k-1}\left(K_{s, t}\right)$ be the graph obtained by replacing an edge of $K_{s, t}$ with a path $P_{2 k+1}$, where $k \geq 2$. Clearly, the odd girth of $S_{2 k-1}\left(K_{s, t}\right)$ is $2 k+3$. Lin, Ning and $\mathrm{Wu}[20]$ showed that the graph $S_{1}\left(K_{\left\lceil\frac{n-1}{2}\right\rceil,\left\lfloor\frac{n-1}{2}\right\rfloor}\right)$ among all nonbipartite triangle-free graphs has maximum spectral radius. Moreover, they posed the following general question:

Question 1 Which class of graphs attain the maximum spectral radius among all nonbipartite graphs with order $n$ and odd girth at least $2 k+3$ ?

Recently, Lin and Guo [20] answer this question. In fact, the proved that the graph $S_{2 k-1}\left(K_{\left\lceil\frac{n-2 k+1}{2}\right\rceil,\left\lfloor\frac{n-2 k+1}{2}\right\rfloor}\right)$ has maximum spectral radius among all graphs of order $n$ whose length of the shortest odd cycle is $2 k+3$.

The secound problem is about the number of odd cycles in a graph of order $n$. There is no explicit formula for calculating the parameter $c_{o}(G)$, the number of odd cycles, in an arbitrary graph. However, there are some bounds and spectral characterizations for this parameter. The signature $s(G)$ of $G$ is the difference between the number of positive eigenvalues and the number of negative eigenvalues of the adjacency matrix $A(G)$. Ma, Yang and Li [22] proved that $c_{o}(G) \geq|s(G)|$. Then, Ma, Wong and Tian [21] characterized graphs whose number of odd cycles equals $|s(G)|$; see [21, Theorems 3.9 and 4.1] for more details. Hare [8] gives some tools for counting odd cycles in graphs, too. For any 2connected nonbipartite graph $G$, it is shown that the cyclomatic number of $G$ is a sharp lower bound for the number of its odd cycles. Also, it is proved that any 3-connected nonbipartite graph $G$ with $n$ vertices contains at least $2 r(G)-2 \geq n$ odd cycles.

Wang and Ma [24] proved the following lower bound for the energy of a graph. 
Theorem 8.( [24, Theorem 4.2]) Let $G$ be a graph with vertex cover number $\beta$. Then $\mathcal{E}(G) \geq 2 \beta-2 c_{o}(G)$. In particular, if $G$ is a bipartite graph, then $\mathcal{E}(G) \geq 2 \beta$.

Remark 9. The lower bound given by Ma and Wang is not suitable for some graphs. For example:

(i) The eigenvalues of the adjacency matrix of the complete graph $K_{n}$ are

$$
\{n-1, \underbrace{-1,-1, \ldots,-1}_{n-1 \text { times }}\}
$$

and so, $\mathcal{E}\left(K_{n}\right)=2 n-2$. On the other hand, $2 \beta-2 c_{o}\left(K_{n}\right)=2(n-1)-2 c_{o}(G)$ which is a trivial lower bound for $\mathcal{E}\left(K_{n}\right)$. So, the lower bound in Theorem 8 is trivial.

(ii) Start with a triangle. Add to each of its edges one new vertex and connect this new vertices to forming a new triangle. Let $G$ be a constructed graph by this method. This graph has $c_{o}(G)=8$ odd cycles and its vertex cover number is $\beta=3$. So, again the lower bound for energy, given in Theorem 8 , is a negative integer.

However, this lower bound is suitable for many other graphs.

Using Theorems 5 and 8 , we have the following immediate corollary which determines a lower bound for the energy of graph in terms of number of edges and maximum degree.

Corollary 10. Let $G$ be a graph with $m$ edges, $c_{o}$ odd cycles and maximum degree $\Delta$. Then

$$
\mathcal{E}(G) \geq \sqrt{(2 \Delta-1)^{2}+8 m}-2\left(\Delta+c_{o}(G)\right)+1 .
$$

In partucular, if $G$ is a bipartite graph, then

$$
\mathcal{E}(G) \geq \sqrt{(2 \Delta-1)^{2}+8 m}-(2 \Delta-1) .
$$

Lemma 11. Let $G=G_{1} \cup G_{2} \cup \cdots \cup G_{s}$, where $G_{1}, G_{2}, \ldots, G_{s}$ are connected components of $G$. Then

$$
\mathcal{E}(G)=\sum_{i=1}^{s} \mathcal{E}\left(G_{i}\right) ; \quad \beta(G)=\sum_{i=1}^{s} \beta\left(G_{i}\right) .
$$

Proof. The first equality follows from this fact that the adjacency matrix of the graph $G$, the union of some graphs $G_{i}$, is a block matrix whose blocks are the adjacency matrices of graphs $G_{i}$, and so the spectrum of $G$ is the union of spectrum of $G_{i}$ s. The secound one follows from this fact that a minimum vertex cover of $G$ is the union of some minimum vertex covers of $G_{i} \mathrm{~s}$. 
Now, as an application of Theorem 2, we find a lower bound for the energy of a graph. From Theorem 2, Corollary 10, and Lemma 11, we have the following immediate corollary for not necessary connected graphs.

Corollary 12. Let $G$ be a graph with $m$ edges, vertex cover number $\beta, k$ odd cycles and maximum degree $\Delta \geq 2$. Then

$$
\mathcal{E}(G) \geq 2(\beta-k) \geq \sqrt{(2 \Delta-1)^{2}+8 m}-2(\Delta+k)+1 .
$$

\section{References}

[1] S. Akbari, M. Ghahremani, M. A. Hosseinzadeh, S. K. Ghezelahmad, H. Rasouli, A. Tehranian, A lower bound for graph energy in terms of minimum and maximum degrees, MATCH Commun. Math. Comput. Chem. 86 (2021) 549-558.

[2] S. Akbari, A. H. Ghodrati, M. A. Hosseinzadeh, Some lower bounds for the energy of graphs, Lin. Algebra Appl. 591 (2020) 205-214.

[3] E. Andrade, J. R. Carmona, G. Infante, M. Robbiano, A lower bound for the energy of hypoenergetic and non hypoenergetic graphs, MATCH Commun. Math. Comput. Chem. 83 (2020) 579-592.

[4] G. Caporossi, D. Cvetković, I. Gutman, P. Hansen, Variable neighborhood search for extremal graphs. 2. Finding graphs with extremal energy, J. Chem. Inf. Comput. Sci. 39 (1999) 984-996.

[5] D. Cvetković, P. Rowlinson, S. Simić, An Introduction to the Theory of Graph Spectra, Cambridge Univ. Press, Cambridge, 2010.

[6] I. Dinur, S. Safra, On the hardness of approximating minimum vertex cover, Ann. Math. 162 (2005) 439-485.

[7] K. C. Das, I. Gutman, Upper bounds on distance energy, MATCH Commun. Math. Comput. Chem. 86 (2021) 611-620.

[8] Donovan R. Hare, Tools for counting odd cycles in graphs, J. Comb. Theory B 139 (2019) 163-192.

[9] S. Filipovski, R. Jajcay, New upper bounds for the energy and spectral radius of graphs, MATCH Commun. Math. Comput. Chem. 84 (2020) 335-343.

[10] C. Godsil, G. Royle, Algebraic Graph Theory, Springer, New York, 2001. 
[11] J. Gu, P. Guo, PEAVC: An improved minimum vertex cover solver for massive sparse graphs, Engin. Appl. Artif. Intell. 104 (2021) 1-14.

[12] I. Gutman, Bounds for total $\pi$-electron energy, Chem. Phys. Lett. 24 (1974) 283-285.

[13] I. Gutman, The energy of a graph, Ber. Math.-Stat. Sekt. Forschz. Graz 103 (1978) 1-22.

[14] D. S. Hochbaum, Efficient bounds for the stable set, vertex cover and set packing problems, Discr. Appl. Math. 6 (1983) 243-254.

[15] A. Jahanbani, J. Rodriguez Zambrano, Koolen-Moulton-type upper bounds on the energy of a graph, MATCH Commun. Math. Comput. Chem. 83 (2020) 497-518.

[16] G. Karakostas, A better approximation ratio for the vertex cover problem, $A C M$ Trans. Alg. 5 (2009) \#41.

[17] F. Kuhn, M. Mastrolilli, Vertex cover in graphs with locally few colors, Inf. Comput. 222 (2013) 265-277.

[18] X. Li, Y. Shi, I. Gutman, Graph Energy, Springer, New York, 2012.

[19] H. Lin, H. Guo, A spectral condition for odd cycles in non-bipartite graphs, Lin. Algebra Appl. 631 (2021) 83-93.

[20] H. Q. Lin, B. Ning, B. Wu, Eigenvalues and triangles in graphs, Comb. Probab. Comput. 30 (2021) 258-270.

[21] X. Ma, D. Wong, F. Tian, Characterization of graphs whose signature equals the number of odd cycles, Lin. Algebra Appl. 11 (2016) 259-273.

[22] H. Ma, W. Yang, S. Li, Positive and negative inertia index of a graph, Lin. Algebra Appl. 438 (2013) 331-341.

[23] B. J. McClelland, Properties of the latent roots of a matrix: the estimation of $\pi$ electron energies, J. Chem. Phys. 54 (1971) 640-643.

[24] L. Wang, X. Ma, Bounds of graph energy in terms of vertex cover number, Lin. Algebra Appl. 517 (2017) 207-216.

[25] D. B. West, Introduction to Graph Theory, Prentice Hall, Upper Saddle River, 2001. 